\title{
The Cl-isotopic composition of lunar KREEP
}

FRANCIS M. MCCUBBIN ${ }^{1}$ AND JESSICA J. BARNES ${ }^{2}$

${ }^{1}$ NASA Johnson Space Center, Mailcode XI, 2101 NASA

Parkway, Houston, TX 77058, USA

${ }^{2}$ Lunar and Planetary Laboratory, University of Arizona, 1629

E University Blvd, Tucson, AZ 85721, United States

We conducted in-situ $\mathrm{Cl}$ isotope measurements of apatite within intercumulus regions and within a holocrystalline olivine-hosted melt inclusion in magnesian-suite troctolite 76535 from Apollo 17 using nanoscale secondary ion mass spectrometry. Magnesian suite troctolite 76535 was collected as a rake sample at station 6 during the Apollo 17 mission and is pristine with minimal evidence of post-crystallization impact processing. These data were collected to place constraints on the Cl-isotopic composition of the last liquid to crystallize from the lunar magma ocean (i.e., urKREEP, named after its enrichments in incompatible lithophile trace elements like potassium, rare earth elements, and phosphorus).

The apatites within 76535 exhibit a limited range in their F-Cl-OH compositions, with most of the variation occurring along the $\mathrm{F}-\mathrm{Cl}$ join. The $\mathrm{F} / \mathrm{Cl}$ ratio of the apatite within the holocrystalline olivine-hosted melt inclusions overlaps with the highest $\mathrm{F} / \mathrm{Cl}$ ratio of apatite within the intercumulus apatite. The apatite in the olivine-hosted melt inclusion and within the intercumulus regions of the sample yielded Clisotopic compositions of $28 \pm 1 \%(2 \sigma)$ and $300 \pm 1 \%$ ( $2 \sigma)$, respectively. The similarity of these values from both textural regimes analyzed indicates that the $\mathrm{Cl}$-isotopic compositions likely represent those of the KREEP-rich magnesian-suite parental magmas. Based on the age of 76535 , these results imply that the KREEP reservoir attained a Cl-isotopic composition of $28-30 \%$ by at least $4.31 \mathrm{Ga}$, consistent with the onset of $\mathrm{Cl}$-isotopic fractionation at the time of lunar magma ocean crystallization or shortly thereafter.

The presence of KREEP-rich olivine-hosted melt inclusions within one of the most pristine and ancient KREEP-rich rocks from the Moon provides a new opportunity to characterize the geochemistry of KREEP. In particular, a broader analysis of stable isotopic compositions of highly and moderately volatile elements could provide an unprecedented advancement in our characterization of the geochemical composition of the KREEP reservoir and of volatile-depletion processes during magma ocean crystallization. 